\title{
Broadband wide-angle dispersion measurements: Instrumental setup, alignment, and pitfalls
}

\author{
A. Farhang, ${ }^{1}$ B. Abasahl, ${ }^{1}$ S. Dutta-Gupta, ${ }^{1}$ A. Lovera,${ }^{1}$ P. Mandracci, ${ }^{2}$ E. Descrovi, ${ }^{2}$ \\ and O. J. F. Martin ${ }^{1}$ \\ ${ }^{1}$ Nanophotonics and Metrology Laboratory, EPFL, 1015 Lausanne, Switzerland \\ ${ }^{2}$ Department of Applied Science and Technology, Politecnico di Torino, 10129 Torino, Italy
}

(Received 6 November 2012; accepted 3 March 2013; published online 19 March 2013)

\begin{abstract}
The construction, alignment, and performance of a setup for broadband wide-angle dispersion measurements, with emphasis on surface plasmon resonance (SPR) measurements, are presented in comprehensive detail. In contrast with most SPR instruments working with a monochromatic source, this setup takes advantage of a broadband/white light source and has full capability for automated angle vs. wavelength dispersion measurements for any arbitrary nanostructure array. A cylindrical prism is used rather than a triangular one in order to mitigate refraction induced effects and allow for such measurements. Although seemingly simple, this instrument requires use of many non-trivial methods in order to achieve proper alignment over all angles of incidence. Here we describe the alignment procedure for such a setup, the pitfalls introduced from the finite beam width incident onto the cylindrical prism, and deviations in the reflected/transmitted beam resulting from the finite thickness of the sample substrate. We address every one of these issues and provide experimental evidences on the success of this instrument and the alignment procedure used. (c) 2013 American Institute of Physics. [http://dx.doi.org/10.1063/1.4795455]
\end{abstract}

\section{INTRODUCTION}

SPR (surface plasmon resonance) spectroscopy has proven to be an extremely useful tool in both biosensing and in aiding the real time tracking of minute changes in biological and chemical processes. ${ }^{1-10}$ Traditional SPR spectroscopy, which measures the absorption of light at resonance via total internal reflection (TIR) excitation of SPPs (surface plasmon polaritons), relies on the use of a monochromatic laser source for illumination of a metallic film, typically $\mathrm{Au}$, on a glass substrate at a specific angle of incidence, where the momentum of incident light is matched to that of the SPR. ${ }^{11-13}$ For example, this momentum match can be obtained for a thin $\mathrm{Au}$ film by placing it onto a glass prism and illuminating via TIR from the glass side in what is known as the Kretschmann configuration. ${ }^{11,14}$ A decrease in reflection then indicates the absorption of light and hence excitation of the surface plasmon. The SPR, being extremely sensitive to the surrounding environment, can then be used to detect minute changes in chemical and biological processes occurring directly at the film/sensor surface. ${ }^{15-21}$ With a monochromatic laser source, one can quantify these processes by tracking variations in the reflection intensity, changes in the incidence angle, or changes in phase. ${ }^{3}$ Further use of a broadband white light source instead of a monochromatic source then allows for tracking of these processes via a detectable change in the SPR wavelength. ${ }^{22}$ Moreover, use of a broadband source eliminates the need for a fine angular scan/search needed to otherwise locate the resonance and allows for full dispersion measurements ${ }^{23-25}$ (angle vs. wavelength) of almost any micro/nanostructure array.

Use of a broadband source and a standard triangular prism, however, only allows for truly accurate measurements at the angle where the source is normally incident to the prism surface. At any other incidence angle, the beam refracts and impinges upon the Au film over a continuum of angles. In order to mitigate this problem, one may instead make use of a half cylinder. ${ }^{26-32}$ So long as one can achieve a precise alignment, the center of the incoming collimated beam will always be normal to the curved surface of the cylindrical prism no matter what the angle of incidence and thus refraction induced splitting of the beam into different spectral components can be eliminated to a great degree. The physical limitation to this, of course, is that the incident beam width is not infinitely small, thus all but the center of the beam will be incident slightly off normal to the cylindrical prism. This will of course cause some horizontal spreading and spectral splitting of the beam. One can reduce these effects greatly by passing the beam through a pinhole to obtain a narrower beam width. Care must also be taken to ensure that the pinhole is not so small as to induce diffraction effects. Furthermore, due to the finite width of the beam, it will be focused onto the Au film by the curved surface of the prism and then subsequently defocused and widened/spread horizontally upon exit. This means that for any given incidence, the sample itself is not being illuminated at just one angle, but rather over a small, but finite, range of angles. In fact, most setups employing a cylindrical prism use this focusing to obtain a smaller beam spot. For SPR sensing where the sensing area is quite small and only a single incidence angle is used, this can be beneficial, however, for wide-angle scans where dispersion curves are to be measured for any arbitrary structural array, this is detrimental. It is important to note, however, that rays at the center of the beam will always be incident normal to the curved surface and thus will fall at the center of the outgoing beam. One can therefore just measure the spectrum of the reflected or transmitted light at the center of the beam. This can be 

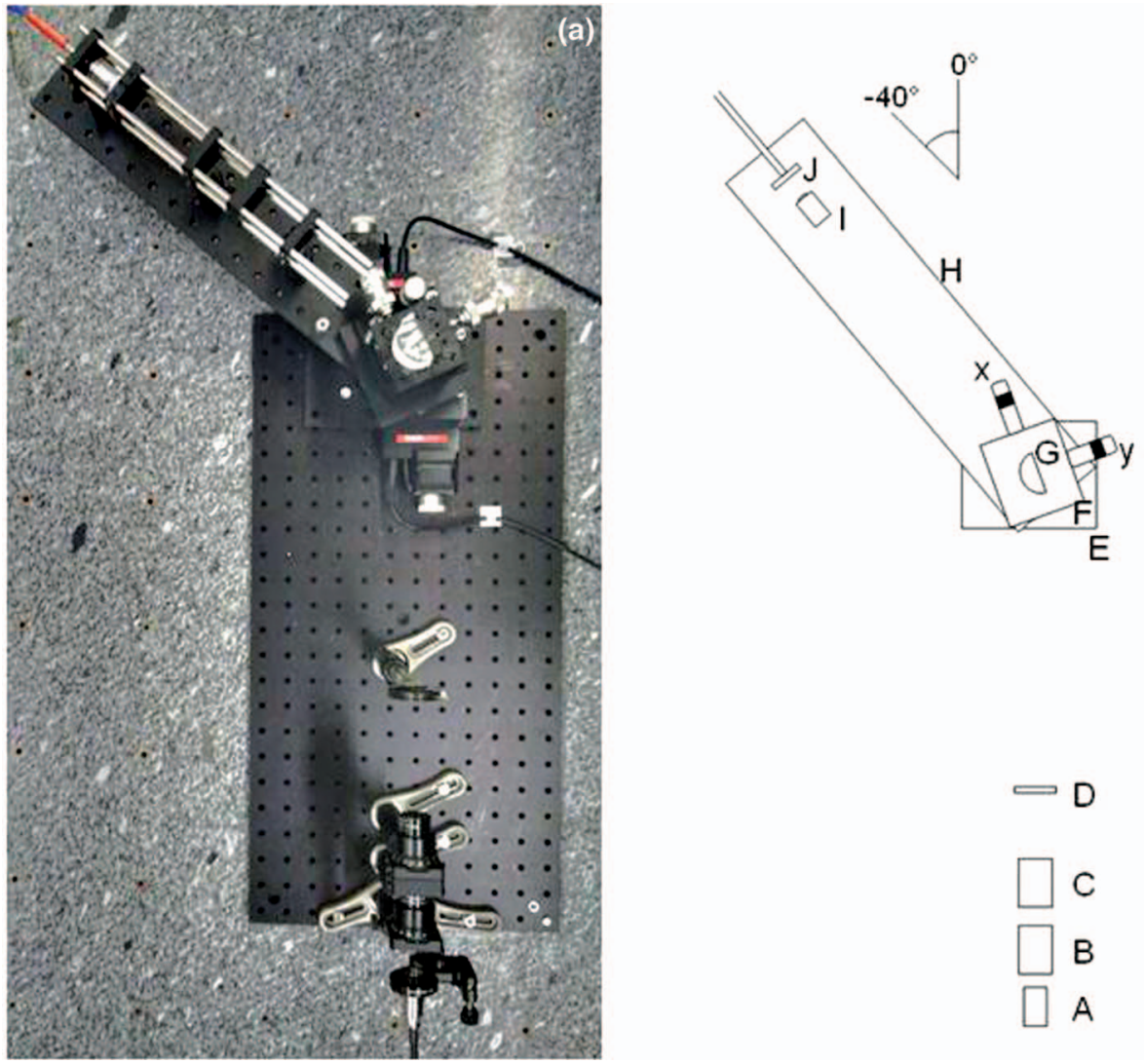

(b)

FIG. 1. (a) Top view of the experimental setup and (b) schematic of the experimental setup when set to a reflection angle of $70^{\circ}$, A: broadband collimated white light source fixed to a kinematic mount for controlling the horizontal and vertical tilt, B: linear polarizer for controlling the output power, C: linear polarizer for controlling the output polarization, D: adjustable pinhole, E: two stepper motors, F: x-y translational stage, G: cylindrical $\mathrm{SiO}_{2}$ prism, $\mathrm{H}$ : detector arm, I: focusing objective, and $\mathrm{J}$ : multimode optical fiber routing the collected light to a spectrometer.

accomplished by placing the collecting component, such as an objective focusing light into an optical fiber, at a distance from the prism where the light has undergone a much greater horizontal spread. Such placement will allow not only for a much higher degree of angular accuracy, but will also eliminate spectral broadening introduced by the undesired angular spread of the incident light.

Alignment of such a setup, though, is less trivial than one may expect. In fact, in order to ensure that the measured spectrum is at a single angle of incidence and that the setup is properly aligned for all angles of incidence, three main criteria must be met. One must ensure that the prism is correctly centered on the axis of rotation, that the beam is well centered on the prism, and that the zero angles of the rotating stage and detector arm are correctly matched. Ensuring all of these alignment criteria simultaneously is in fact possible, however, it requires the use of a few clever techniques. In this article we will not only provide a step by step account on how to carry out this crucial alignment, but also highlight additional pitfalls introduced by mounting samples with a finite substrate thickness onto the prism. Results are then presented in reflection comparing the measured dispersion of the SPP mode of an $\mathrm{Au}$ film to those found by analytical transfer matrix method calculations in order to demonstrate the accuracy of the alignment procedure. Results showing excellent agreement for additional multilayer film structures are also presented in order to highlight the versatility and accuracy of the instrument.

\section{EXPERIMENTAL SETUP}

\section{A. Components and configuration}

An image and schematic of the experimental setup considered in this article is shown in Figs. 1(a) and 1(b). The setup consists of a collimated broadband light source (here a SC400 WhiteLase Supercontinuum Source from Fianium) fixed onto a Thorlabs Kinematic Mount (KM100x), two linear polarizers to control the beam intensity and polarization, an adjustable $0.5-5 \mathrm{~mm}$ iris/pinhole for beam width reduction, two NR360 stepper motors from Thorlabs, one for controlling the rotation of a mounted $\mathrm{x}-\mathrm{y}$ translational stage on which a cylindrical prism (half cylinder of $\mathrm{SiO}_{2}$ ) is centered and another to control the rotation of the detector arm on which sits an optical objective focusing light into an optical fiber that is routed to an Ocean Optics HR4000-CG-UE-NIR spectrometer. The objective (20/0.35) and fiber (Ocean Optics QP200-2-VIS-NIR) are mounted onto a cage system centered along the length of the detection arm in order to ensure an easy and proper alignment with the incoming beam. The entire system is controlled and automated via LabVIEW software. The stage controls the rotation of the prism, while the arm controls the rotation of the focusing objective and collecting fiber. Note that we refer to a clockwise rotation of the stage or detector arm by a positive angle $\theta$ and set the reference $\theta=0^{\circ}$ to be that at which the detector arm is aligned in the vertical direction, i.e., parallel to the source, and the stage is oriented 


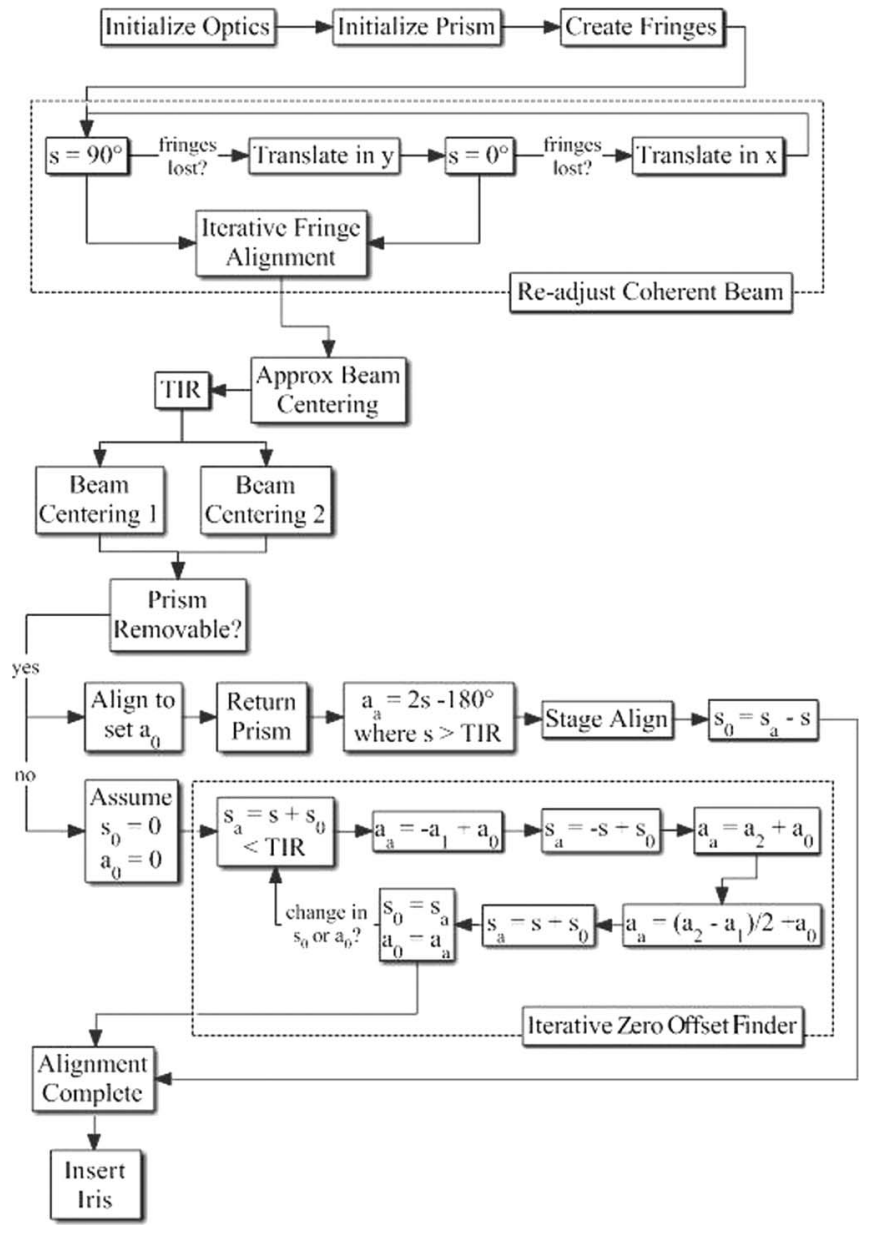

FIG. 2. Flow chart outlining the full alignment procedure for the experimental setup shown in Fig. 1.

so that the $\mathrm{x}$ and $\mathrm{y}$ translation knobs are aligned along the horizontal and vertical directions, respectively. Based on this convention, we can see that with the stage rotated to an angle of $\mathrm{s}=70^{\circ}$, the detector arm must correspondingly be rotated to an angle $\mathrm{a}=2 \mathrm{~s}-180^{\circ}=-40^{\circ}$ for reflection measurements, Fig. 1(b).

\section{B. Setup and alignment}

Almost every step in the alignment of such a setup is highly dependent on the accuracy of the preceding steps. Therefore, we have included a detailed step-by-step process for the alignment. The process is outlined as a flow chart in Fig. 2 and details for each step of the flow chart are given in Table I. The process consists of four major steps. First is the initialization of the incident polarization state and power of the source beam, which must also be made parallel to the working plane. Second and most non-trivial is the centering of the prism on the axis of rotation. It is crucial to ensure correct centering of the prism for any of the subsequent alignment steps to be meaningful. Third is the centering of the beam onto the axis of rotation/center of the prism. Fourth and final is the initialization of the stage and detector arm to zero. This will ensure correct alignment of the detector arm with the reflected or transmitted beam and incidence of the incoming beam onto the prism at the intended angle of incidence. Definitions for variables used in the flow chart of Fig. 2 are as follows: "a" and "s" denote the angles of the detector arm and stage as displayed by the controlling software before they are corrected to zero via zero offset angles " $\mathrm{a}_{0}$ " and " $\mathrm{s}_{0}$ ", " $\mathrm{a}_{\mathrm{a}}$ " and " $\mathrm{s}_{\mathrm{a}}$ " denote the corrected angles of the detector arm and stage after the addition of the zero offsets: $a_{a}=a$ $+\mathrm{a}_{0}$ and $\mathrm{s}_{\mathrm{a}}=\mathrm{s}+\mathrm{s}_{0}$. Additionally, angles $\mathrm{s}$ and $\mathrm{a}$ are at times denoted with subscripts $s_{1}, s_{2}$ or $a_{1}, a_{2}$ for steps requiring rotation to multiple angles in order to complete the alignment.

\section{CORRECTING FOR SUBSTRATE INDUCED BEAM DEVIATIONS}

Based on the alignment steps made, measurements for light totally internal reflected from the flat face of the prism simply require that the detector arm be rotated to an angle $a_{a}$ $=\mathrm{a}_{0}+2 \mathrm{~s}-180^{\circ}$ when the stage/prism is rotated to an angle $\mathrm{s}_{\mathrm{a}}=\mathrm{s}+\mathrm{s}_{0}$. Similarly, via Snell's law, transmission measurements for the same stage angle $s_{a}=s+s_{0}$ below the TIR angle require the detector arm to be set to an angle $\mathrm{a}_{\mathrm{a}}=\mathrm{a}_{0}+$ $\mathrm{s}-\sin ^{-1}(\mathrm{n} \times \sin (\mathrm{s}))$.

Most often, however, the structures being optically investigated will not reside directly on the flat face of the prism. Rather, they will often lie on a glass substrate attached and optically coupled to the prism via index matching gel. Alternatively it may be possible/preferred to place the sample in a reversed orientation onto the prism, such that the structures are in direct contact with the thin layer of index matching gel. Of course in the latter case, almost no corrections need to be made in the reflection or transmission collection angle of the detector arm, so long as the index matching gel layer is very thin. In the former case, corrections for the angle of the detector arm are needed for both reflection and transmission measurements. The amount of correction necessary depends not only on the thickness of the substrate, but also on the distance 1 between the prism and focusing objective, the index of the prism and substrate, and for reflection measurements the radius $r$ of the prism. Assuming use of a substrate with a thickness $\mathrm{t} \ll \mathrm{r}$ and a refractive index of the substrate $\mathrm{n}$ very close or equal to that of the prism, one can via simple geometry, Snell's Law, and trigonometric simplifications, derive the corrected angle of the detector arm necessary to directly intersect the reflected beam:

$$
a_{a}=a_{0}+2 s-180+\tan ^{-1}\left[\tan \left(\sin ^{-1}\left(\frac{b}{r}\right)-\sin ^{-1}\left(n \cdot \frac{b}{r}\right)\right)+\frac{b}{l}\right] \Rightarrow\left\{\begin{array}{l}
s<45^{\circ} \Rightarrow b=\frac{t \sin (2 s)}{\cos (s)} \\
s \geq 45^{\circ} \Rightarrow b=\frac{t \sin (180-2 s)}{\cos (s)} .
\end{array}\right.
$$


TABLE I. Details for the alignment steps shown in the flow chart of Fig. 2.

Flow chart steps and details

Initialize optics: Set the desired polarization and output power of the source and align it such that the beam propagates parallel to the working plane and is vertically level with the focusing objective.

Initialize prism: Place the prism onto the center of the stage and set the stage angle as close to zero as best as can be done by visual inspection.

Create fringes: Use an additional light source that is coherent, such as a laser or white light supercontinuum source. Direct the beam so that it barely grazes the circular face of the prism. The small portion of the beam grazing and reflecting off of the prism will coherently interfere with the rest of the beam. A screen placed in front of the two interfering parts of the beam will then show the immerging fringe pattern.

Translate in $\mathbf{x}$ or $\mathbf{y}$ : In order to regain any fringes lost during rotation of the stage (see next step), the stage can be translated along the $\mathrm{x}$-axis or $\mathrm{y}$-axis (via the $\mathrm{x}$ and $\mathrm{y}$ translation knobs shown in Fig. 1(b)) for stage angles $\mathrm{s}=0^{\circ}$ and $\mathrm{s}=90^{\circ}$, respectively.

Iterative fringe alignment: Once fringes are visible at both $\mathrm{s}=0^{\circ}$ and $\mathrm{s}=90^{\circ}$, $\mathrm{s}$ should be alternatively rotated between $0^{\circ}$ and $90^{\circ}$ and the stage should be iteratively translated along $\mathrm{x}$ and $\mathrm{y}$ until the same fringe pattern is seen for all angles between $\mathrm{s}=0^{\circ}$ and $90^{\circ}$. This ensures that the prism is well centered along the axis of rotation.

Re-adjust coherent beam: In the case that a fringe pattern is lost when rotating from $\mathrm{s}=0^{\circ}$ to $\mathrm{s}=90^{\circ}$, it is very likely that the prism is too far offset from the center of rotation. Thus a better first guess is needed for centering the prism before readjusting the coherent beam and obtaining an interfering fringe pattern at both $\mathrm{s}=0^{\circ}$ and $\mathrm{s}=90^{\circ}$. Additionally, if a convergence to a single fringe pattern for all $\mathrm{s}=0^{\circ}-90^{\circ}$ cannot be reached, then it is likely that the coherent beam's grazing trajectory onto the prism needs to be slightly adjusted before recontinuing with the iterative fringe adjustment step.

Approximate beam centering: Adjust the horizontal tilt of the white light source such that it is incident approximately on the center of the prism.

TIR: Rotate the stage till total internal reflection of the incident beam is observed.

Beam centering 1: So long as the source provides enough power, both a primary beam spot and a secondary beam spot (due to secondary reflections from the curved exit facet of the prism) should be visible on the incident surface of the prism. Adjust the horizontal tilt of the source beam till the two spots are exactly superimposed. The beam is now well centered on the prism.

Beam centering 2: If the beam is too weak to see two spots, place an adjustable iris set to a size slightly larger than that of the beam diameter, so that the incident beam passes directly through the center of the opening. Direct reflection of the incident beam from curved air-prism interface will result in a horizontally stretched beam visible on the iris. Adjust the horizontal tilt of the beam till this stretched beam shows arms of equal widths with respect to the center of the iris. Care should be taken to ensure that the beam spot is fully able to pass through the opening of the iris at all times. So long as the iris is placed at a distance of $20 \mathrm{~cm}$ or greater from the prism, good alignment will be insured.

Prism removable?: Can the prism be removed and then be placed back in the exact same position?

Align to set $\mathbf{a}_{\mathbf{0}}$ : Temporarily remove the prism from the stage and adjust the detector arm angle a to an angle $\mathrm{a}_{0}$ at which maximum spectral power is focused into the objective. $\mathrm{a}_{0}=$ zero angle of detection arm.

Stage align: Rotate the stage to an angle $\mathrm{s}_{\mathrm{a}}$ till the reflected and horizontally stretched beam is centered on the detecting objective. Depending on the spectral distribution of the source being used, the prism material, prism radius, and beam width, the beam spot may exhibit maximum spectral power not at the visible center, but may instead exhibit two maxima equidistant from either side of the visible center. If this is the case, take the mean angle at which these spectral maxima are located to be $\mathrm{s}_{\mathrm{a}}$.
TABLE I. (Continued.)

Flow chart steps and details

Iterative zero offset finder: An iterative algorithm for finding the zero offset angles required for aligning the stage and detector arm. Assume zero angles for the stage $\mathrm{s}_{0}=0$ and detector arm $\mathrm{a}_{0}=0$. Rotate the stage to some displayed angle $s$ greater than the true zero angle, but less than that of the TIR angle, set the detector arm at a displayed angle $-a_{1}$ to intersect exactly the transmitted light. Rotate the stage to a displayed angle $-\mathrm{s}$ and again the detector arm to a displayed angle $\mathrm{a}_{2}$ to intersect exactly the transmitted beam. Set the detector arm to an intermediate angle $a=\left(a_{2}-a_{1}\right) / 2$ and then rotate the stage to a displayed angle s such that the transmitted light is incident directly onto the center of the focusing objective of the detecting $\operatorname{arm}$. Set $\mathrm{s}_{0}=\mathrm{s}+\mathrm{s}_{0}$ and $\mathrm{a}_{0}=\mathrm{a}+\mathrm{a}_{0}$. Repeating till no noticeable change is seen in $\mathrm{s}_{0}$ and $\mathrm{a}_{0}$.

Insert iris: In order to reduce spectral broadening and obtain just the fraction of the beam that is incident normal to the curvature of the prism, place an iris directly after the polarizing components. The size of the opening should be the smallest possible without inducing diffraction effects. Move the stage to an angle $\mathrm{s}_{\mathrm{a}}=\mathrm{s}+\mathrm{s}_{0}$ where $\mathrm{s}>$ TIR and the detector arm to an angle $\mathrm{a}_{\mathrm{a}}=\mathrm{a}_{0}+2 \mathrm{~s}-180$. Adjust the position of the iris to transmit just the light which falls directly into the center of the objective. This will ensure that only light normal to the prisms curvature is detected.

Similarly in transmission, an expression for the angle of the detector arm can be easily derived for incidence below the TIR angle. Here both the index of the prism $n_{p}$ and substrate $\mathrm{n}_{\mathrm{s}}$ are taken into account, resulting in a corrected detector arm angle:

$$
\begin{aligned}
a_{a}= & a_{0}+s-\sin ^{-1}\left(n_{p} \sin (s)\right) \\
& +\sin ^{-1}\left[\frac{t \cdot n_{p} \sin (s)}{l} \cdot\left(1-\frac{\sqrt{1-n_{p}^{2} \sin ^{2}(s)}}{\sqrt{n_{s}^{2}-n_{p}^{2} \sin ^{2}(s)}}\right)\right] .
\end{aligned}
$$

Using the above equations, we calculate the angular corrections that must be made in reflection or transmission across a wide range of incidence angles for substrate thicknesses $\mathrm{t}=0-0.5 \mathrm{~mm}$, the angular corrections being the last terms in Eqs. (1) and (2). We use the following parameters from the setup shown in Fig. 1: $\mathrm{r}=20 \mathrm{~mm}, 1=300 \mathrm{~mm}, \mathrm{n}_{\mathrm{s}}=1.55$, and $n_{p}=1.46$. The results shown in Figs. 3(a) and 3(b) indicate that for measurements in reflection, angular deviations can be quite substantial even at just $\mathrm{s}=20^{\circ}$ incidence. In transmission, deviations are not nearly as significant, but can still become noticeable especially if the discrepancy between $\mathrm{n}_{\mathrm{s}}$ and $\mathrm{n}_{\mathrm{p}}$ becomes significant. Thus, it is evident that in such a system, substrate induced angular deviations must be taken into account.

\section{EXPERIMENTAL VERIFICATION AND VERSATILITY}

In order to demonstrate the ability of this system to measure full dispersions of any desired system, we show as a first example reflection spectra angular scans made for a $50 \mathrm{~nm}$ thick Au film on a fused silica substrate of thickness $0.5 \mathrm{~mm}$ $\left(\varepsilon_{\mathrm{SiO} 2}=2.13\right)$, Fig. 4(a). Note that corrections for substrate induced angular deviations were fully taken into account. Use 

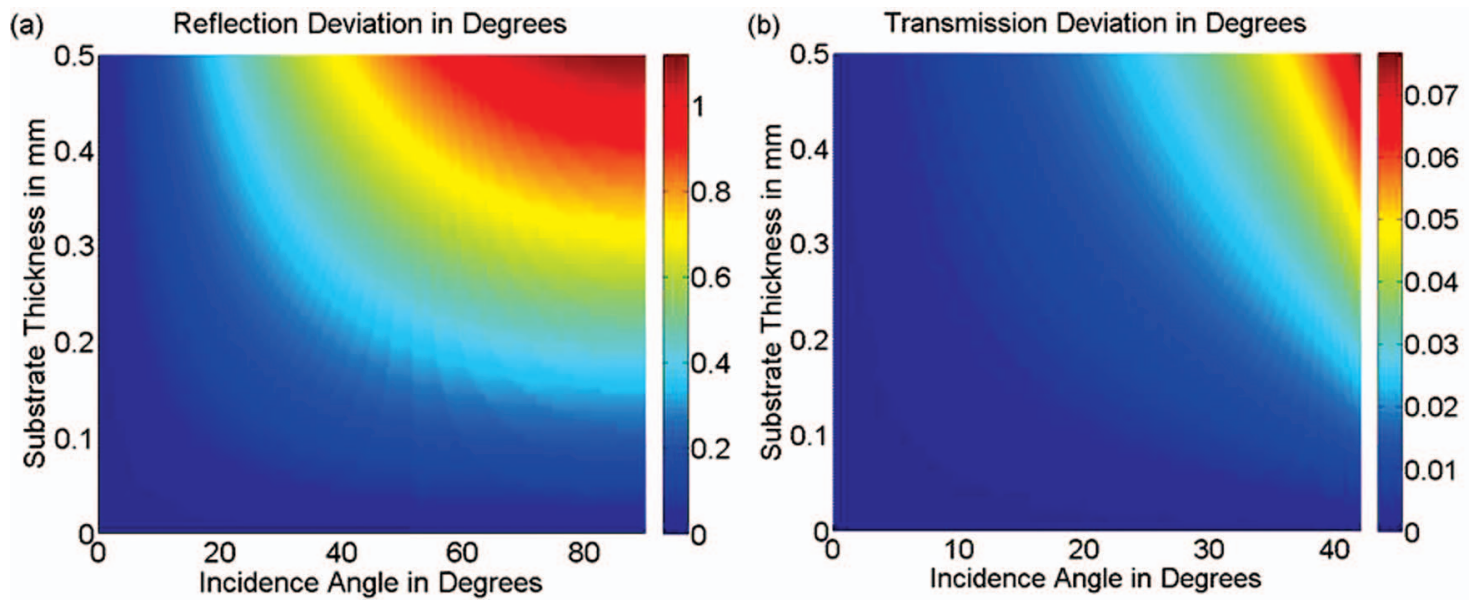

FIG. 3. Angular corrections necessary for the collection angle of the detector arm due to substrate induced angular deviations in (a) reflection and (b) transmission. As can be seen, angular deviations are much more significant in reflection than in transmission, but in both cases grow rapidly with incidence angle and substrate thickness.

of LabVIEW software and the alignment process outlined in Fig. 2 and Table I allow for entirely automated measurements over all angles of incidence in a matter of minutes. As expected, the excitation of a propagating SPP wave at the metal air interface can be clearly seen as a very pronounced dip in the measured reflection spectra. The position of this dip is traced and the result is plotted in Fig. 4(b) along with calculations made via an analytical transfer matrix (TMM) formulation, ${ }^{33}$ where the wavelength dependent permittivity of fused silica ${ }^{34}$ and $\mathrm{Au}^{35}$ were used. As can be seen the two show an extremely good agreement, thereby demonstrating the proper alignment of this system and its capability in obtaining full dispersion measurements both quickly and accurately.

In order to further demonstrate the capabilities of this system and its versatility in measuring the dispersion of any arbitrary system, we show and compare both experimental measurements and simulation results for a bilayer $\mathrm{Au}$ film system (Fig. 5) supporting coupled SPPs and a

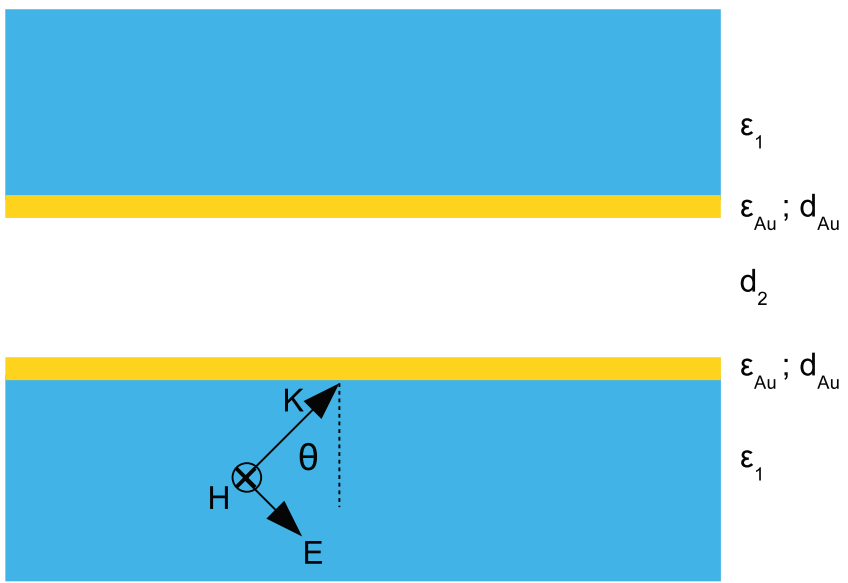

FIG. 5. A bilayer Au film structure with an air gap of thickness of $d_{2}=580$ $\mathrm{nm}$. The Au layers are $\mathrm{d}_{\mathrm{m}}=40 \mathrm{~nm}$ thick. The structure is illuminated with TM polarized light from below at various angles $\theta$ in order to excite SPPs. (a)

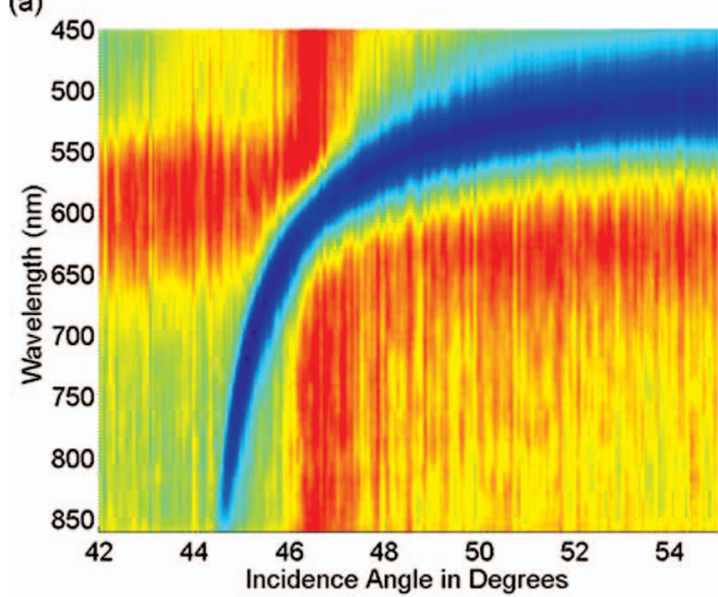

(b)

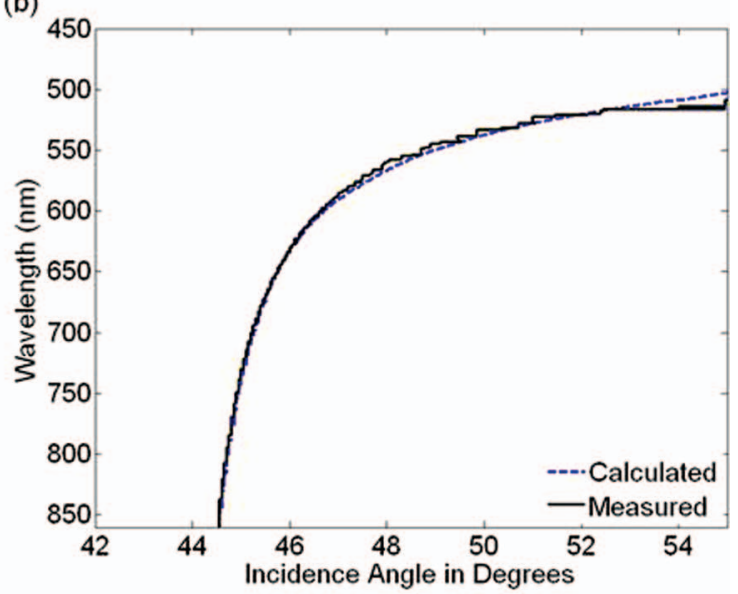

FIG. 4. (a) Measured reflection spectra from a $50 \mathrm{~nm}$ Au film evaporated onto a fused silica/ $\mathrm{SiO}_{2}$ substrate coupled to the glass prism via index matching gel. (b) Comparison of the measured SPP dip to that obtained via an analytical transfer matrix calculation, showing indeed a very good match. 


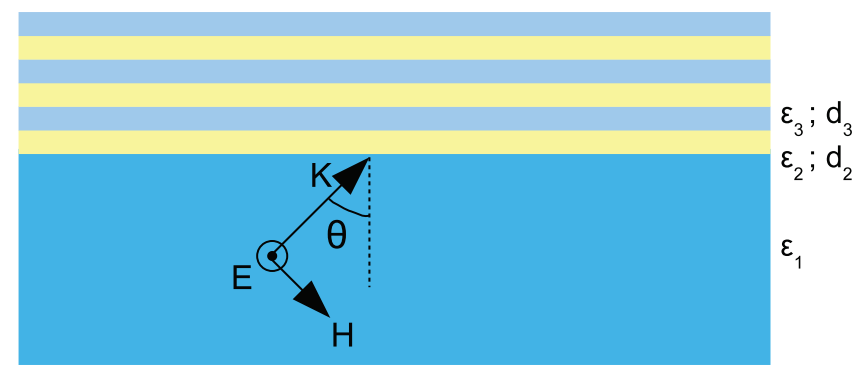

FIG. 6. A multilayer structure composed of three sets of alternating layers of $\mathrm{Si}$ enriched silicon nitride and $\mathrm{SiO}_{2}$ on a float glass substrate. The thickness $d_{2}$ of the silicon nitride layers is $105 \mathrm{~nm}$ and the thickness $\mathrm{d}_{3}$ of the $\mathrm{SiO}_{2}$ layers is $275 \mathrm{~nm}$. The structure is illuminated with TE polarized light from below at various angles $\theta$ in order to excite BSWs.

multilayer structure (Fig. 6), supporting Bloch surface waves (BSWs). ${ }^{36}$

The bilayer Au film system is composed of float glass $\left(\varepsilon_{1}\right.$ $=2.31$ ) substrate and superstrate, both of thickness $0.55 \mathrm{~mm}$. The air gap between the two $\mathrm{d}_{\mathrm{m}}=40 \mathrm{~nm}$ thick Au films is $\mathrm{d}_{2}=580 \mathrm{~nm}$. The bilayer Au film system in Fig. 5 exhibits two spectral curves, one for the symmetric SPP mode and the

(a)

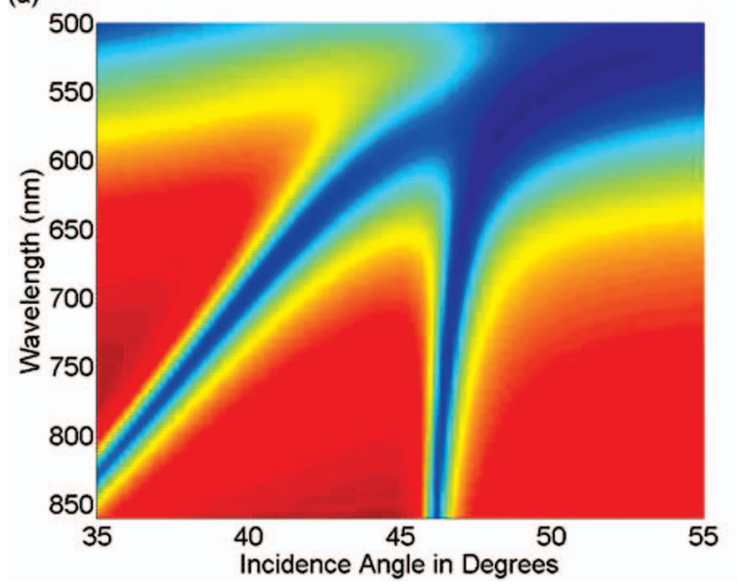

other for the anti-symmetric SPP. The level of coupling between the two modes and thus their spectral separation can either be controlled by varying the spacer thickness or by changing the angle of incidence. TMM simulations for TM excitation result in the reflection spectra shown in Fig. 7(a). A comparison of the simulation to experimental measurements (Fig. 7(b)) shows an excellent agreement.

Finally experimental reflection spectra for the multilayer structure shown in Fig. 6 are presented and compared with TMM simulations. The structure consists of three sets of alternating layers of $\mathrm{Si}$ enriched silicon nitride and $\mathrm{SiO}_{2}$ on a float glass substrate $\left(\varepsilon_{1}=2.31\right)$ of $0.55 \mathrm{~mm}$ thickness. The thickness $\mathrm{d}_{2}$ of the silicon nitride layers is $105 \mathrm{~nm}$ and the thickness $d_{3}$ of the $\mathrm{SiO}_{2}$ layers is $275 \mathrm{~nm}$. The structure is fabricated in the same manner as that described in Ref. 36 and the frequency dependent dielectric data of the silicon nitride $\left(\varepsilon_{2}\right)$ are estimated by the means of standard optical measurements. ${ }^{37}$ The dielectric constant of the alternating $\mathrm{SiO}_{2}$ layers are taken to be $\varepsilon_{3}=2.19$. TMM simulations for TE excitation result in the reflection spectra shown in Fig. 8(a). As can be seen, a shallow and very narrow spectral dip extending from $\lambda=1000 \mathrm{~nm}-500 \mathrm{~nm}$ is present, thereby

(b)

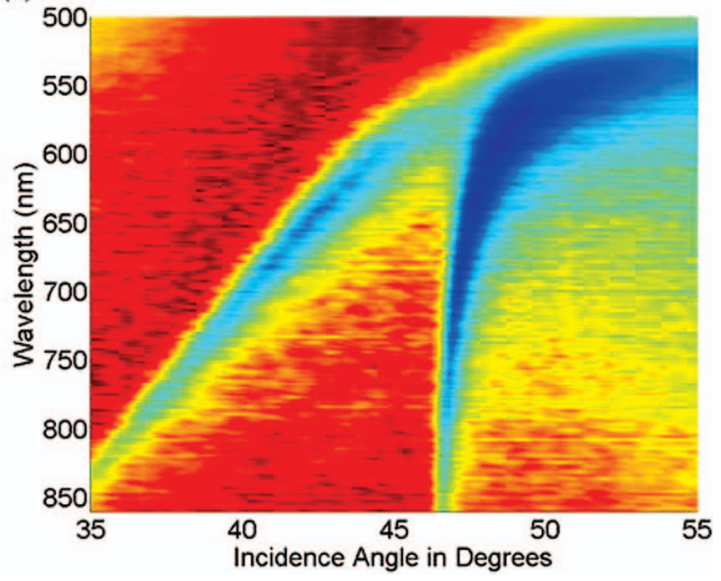

FIG. 7. (a) Simulated reflection spectra of the structure shown in Fig. 5, showing spectral dips of the symmetric and antisymmetric SPPs. (b) Measured reflection spectra for the fabricated structure, showing an excellent agreement between the two.

(a)

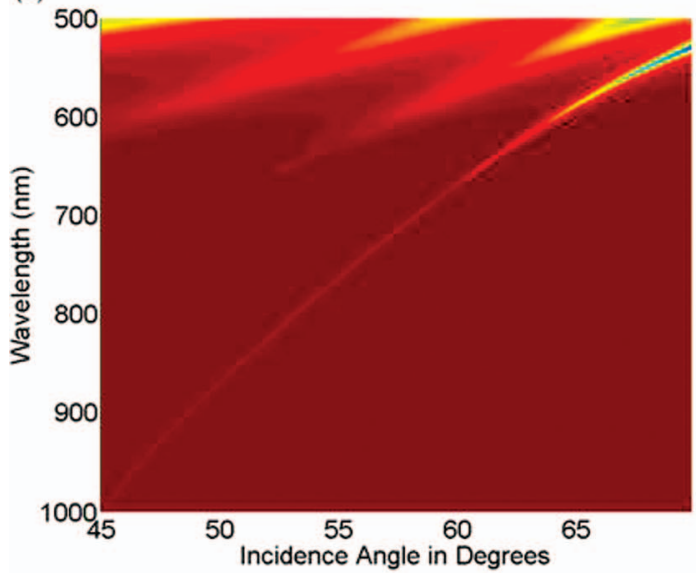

(b)

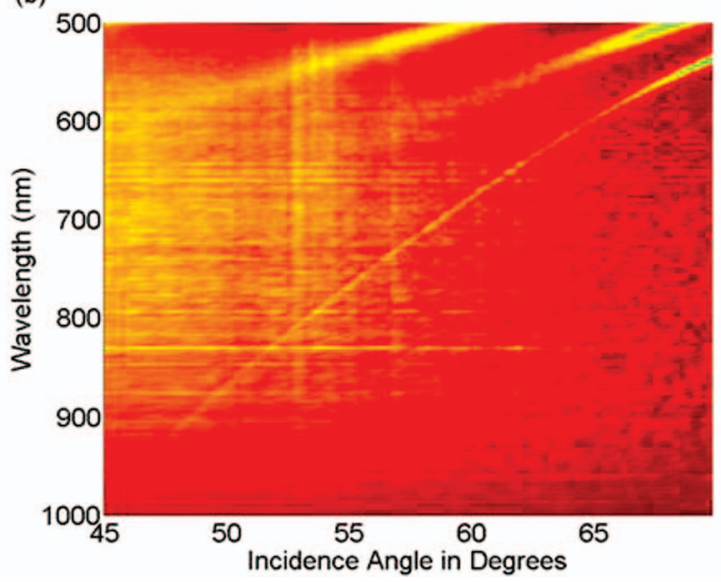

FIG. 8. (a) Simulated reflection spectra for the structure shown in Fig. 6, showing a shallow and very narrow BSW dip extending from $\lambda=1000 \mathrm{~nm}-500 \mathrm{~nm}$. (b) Measured reflection spectra for the fabricated structure, showing an excellent agreement between the two. 
indicating the excitation of a BSW. Note the additional dips seen at lower wavelengths are simply due to guided modes present in the dielectric layers. Just as in the last example, a comparison of simulation and experimental results (Fig. 8(b)) shows excellent agreement, thereby demonstrating the capability of the instrument in measuring not only very narrow angular/spectral features, but also its accuracy and versatility in determining dispersion curves for a wide variety of complex photonic structures.

\section{CONCLUSION}

We have presented detailed accounts on the alignment procedure for an instrument capable of broadband wide-angle dispersion measurements, operating with a cylindrical prism. The system and employed alignment procedure demonstrate extremely accurate angle vs. wavelength dispersion measurements for a $50 \mathrm{~nm}$ Au Film on a $\mathrm{SiO}_{2}$ substrate excited via TIR illumination. Special emphasis was placed on the use of a cylindrical prism rather than a triangular one in order to mitigate refraction effects. Additionally, methods for reducing spectral broadening of the reflected or transmitted light induced by the curved surface of the prism were presented. Finally, the significance of deviations in the reflected/transmitted beam resulting from the finite thickness of the sample substrate were highlighted and accounted for. Besides allowing for extremely accurate measurements of SPP dispersions, the simplicity and robustness of this device make it suitable and desirable for multi-angle spectroscopic studies of any nanostructure or microstructure array, such as multilayers supporting Bloch surface waves, ${ }^{38}$ nanoscale grating/particle structures, ${ }^{39,40}$ or composite metallic nanostructures. ${ }^{41,42}$

\section{ACKNOWLEDGMENTS}

We would like to thank Dr. Andreas Kern for bringing to light a very clever yet simple optical interference technique for centering the cylindrical prism on the axis of rotation. Funding from the State Secretariat for Education and Research SER within the Indo Swiss Joint Research Programme is also gratefully acknowledged.

${ }^{1}$ J. N. Anker, W. P. Hall, O. Lyandres, N. C. Shah, J. Zhao, and R. P. Van Duyne, Nat. Mater. 7, 442-453 (2008).

${ }^{2}$ R. J. Green, R. A. Frazier, K. M. Shakesheff, M. C. Davies, C. J. Roberts, and S. J. B. Tendler, Biomaterials 21, 1823-1835 (2000).

${ }^{3}$ J. Homola, in Surface Plasmon Resonance Based Sensors, edited by J. Homola (Springer, 2006).

${ }^{4}$ J. Homola, Chem. Rev. 108, 462-493 (2008).

${ }^{5}$ Y. H. Huang, H. P. Ho, S. Y. Wu, and S. K. Kong, Adv. Opt. Technol. 2012, (2012).

${ }^{6}$ B. Liedberg, C. Nylander, and I. Lunstrom, Sens. Actuators 4, 299-304 (1983).
${ }^{7}$ M. Piliarik, L. Parova, and J. Homola, Biosens. Bioelectron. 24, 1399-1404 (2009).

${ }^{8}$ A. D. Taylor, J. Ladd, Q. Yu, S. Chen, J. Homola, and S. Jiang, Biosens. Bioelectron. 22, 752-758 (2006).

${ }^{9}$ G. J. Wegner, A. W. Wark, H. J. Lee, E. Codner, T. Saeki, S. Fang, and R. M. Corn, Anal. Chem. 76, 5677-5684 (2004).

${ }^{10}$ W. Zhang, L. Huang, C. Santschi, and O. J. F. Martin, Nano Lett. 10, 10061011 (2010)

${ }^{11}$ E. Kretschmann and H. Raether, Z. Naturforsch. A 23, 2135 (1968).

${ }^{12}$ A. Otto, Z. Phys. 216, 398 (1968).

${ }^{13}$ H. Raether, Physics of Thin Films (Academic, New York, 1977), Vol. 9, pp. 145.

${ }^{14}$ H. Raether, Springer Tracts Mod. Phys. 111, 1-133 (1988).

${ }^{15}$ I. Abdulhalim, M. Zourob, and A. Lakhtakia, Electromagnetics 28, 214 242 (2008).

${ }^{16}$ M. Besenicar, P. Macek, J. H. Lakey, and G. Anderluh, Chem. Phys. Lipids 141, 169-178 (2006).

${ }^{17}$ J. Dostálek and W. Knoll, Biointerphases 3, FD12-FD22 (2008).

${ }^{18}$ J. Ferreira, M. J. L. Santos, M. M. Rahman, A. G. Brolo, R. Gordon, D. Sinton, and E. M. Girotto, J. Am. Chem. Soc. 131, 436-437 (2009).

${ }^{19}$ U. Jonsson, L. Fagerstam, B. Ivarsson, B. Johnsson, R. Karlsson, K. Lundh, S. Lofas, B. Persson, H. Roos, and I. Ronnberg, BioTechniques 11, 620627 (1991).

${ }^{20}$ S. Scarano, M. Mascini, A. P. F. Turner, and M. Minunni, Biosens. Bioelectron. 25, 957-966 (2010).

${ }^{21}$ J. L. Wang, A. Munir, and H. S. Zhou, Talanta 79, $72-76$ (2009).

${ }^{22}$ K. Johansen, H. Arwin, I. Lundstrom, and B. Liedberg, Rev. Sci. Instrum. 71, 3530-3538 (2000).

${ }^{23}$ N.-S. Eum, D.-E. Kim, S.-H. Yeom, B.-H. Kang, K.-J. Kim, C.-S. Park, and S.-W. Kang, Biosystems 98, 51-55 (2009).

${ }^{24}$ Z.-m. Qi, M. Wei, H. Matsuda, I. Honma, and H. Zhou, Appl. Phys. Lett. 90, 181112 (2007).

${ }^{25}$ J. D. Swalen, J. G. Gordon II, M. R. Philpott, A. Brillante, I. Pockrand, and R. Santo, Am. J. Phys. 48, 669-672 (1980).

${ }^{26}$ S. Boussaad, J. Pean, and N. J. Tao, Anal. Chem. 72, 222-226 (2000).

${ }^{27}$ A. Ikehata, X. Li, T. Itoh, Y. Ozaki, and J.-H. Jiang, Appl. Phys. Lett. 83, 2232-2234 (2003).

${ }^{28}$ S. R. Karlsen, K. S. Johnston, R. C. Jorgenson, and S. S. Yee, Sens. Actuators B 25, 747-749 (1995).

${ }^{29}$ A. A. Kolomenskii, P. D. Gershon, and H. A. Schuessler, Appl. Opt. 39, 3314-3320 (2000).

${ }^{30}$ E. Kretschmann, Z. Phys. A: Hadrons Nucl. 241, 313-324 (1971).

${ }^{31}$ M. Morjan, H. Zuchner, and K. Cammann, Surf. Sci. 603, 1353-1359 (2009).

${ }^{32}$ N. J. Tao, S. Boussaad, W. L. Huang, R. A. Arechabaleta, and J. Dagnese, Rev. Sci. Instrum. 70, 4656-4660 (1999).

${ }^{33}$ B. E. A. Saleh and M. C. Teich, in Fundamentals of Photonics (John Wiley \& Sons, Inc., 2001), pp. 238-271.

${ }^{34}$ I. H. Malitson, J. Opt. Soc. Am. 55, 1205-1208 (1965).

${ }^{35}$ P. B. Johnson and R. W. Christy, Phys. Rev. B 6, 4370-4379 (1972).

${ }^{36}$ E. Descrovi, T. Sfez, L. Dominici, W. Nakagawa, F. Michelotti, F. Giorgis, and H.-P. Herzig, Opt. Express 16, 5453-5464 (2008).

${ }^{37}$ C. Ricciardi, V. Ballarini, M. Galli, M. Liscidini, L. C. Andreani, M. Losurdo, G. Bruno, S. Lettieri, F. Gesuele, P. Maddalena, and F. Giorgis, J. Non-Cryst. Solids 352, 1294-1297 (2006).

${ }^{38}$ E. Descrovi, T. Sfez, M. Quaglio, D. Brunazzo, L. Dominici, F. Michelotti, H. P. Herzig, O. J. F. Martin, and F. Giorgis, Nano Lett. 10, 2087-2091 (2010).

${ }^{39}$ A. Christ, O. J. F. Martin, Y. Ekinci, N. A. Gippius, and S. G. Tikhodeev, Nano Lett. 8, 2171-2175 (2008).

${ }^{40}$ K. B. Crozier, E. Togan, E. Simsek, and T. Yang, Opt. Express 15, 1748217493 (2007).

${ }^{41}$ A. Christ, G. Lévêque, O. J. F. Martin, T. Zentgraf, J. Kuhl, C. Bauer, H. Giessen, and S. G. Tikhodeev, J. Microsc. 229, 344-353 (2008).

${ }^{42}$ Y. Chu and K. B. Crozier, Opt. Lett. 34, 244-246 (2009). 